\title{
Effect of gain nonlinearity in semiconductor lasers
}

\author{
Jensen, Niels H.; Christiansen, Peter Leth; Skovgaard, Ove
}

Published in:

Physical Review B

Link to article, DOI:

10.1103/PhysRevB.38.8219

Publication date:

1988

Document Version

Publisher's PDF, also known as Version of record

Link back to DTU Orbit

Citation (APA):

Jensen, N. H., Christiansen, P. L., \& Skovgaard, O. (1988). Effect of gain nonlinearity in semiconductor lasers. Physical Review B, 38(12), 8219-8225. https://doi.org/10.1103/PhysRevB.38.8219

\section{General rights}

Copyright and moral rights for the publications made accessible in the public portal are retained by the authors and/or other copyright owners and it is a condition of accessing publications that users recognise and abide by the legal requirements associated with these rights.

- Users may download and print one copy of any publication from the public portal for the purpose of private study or research.

- You may not further distribute the material or use it for any profit-making activity or commercial gain

- You may freely distribute the URL identifying the publication in the public portal

If you believe that this document breaches copyright please contact us providing details, and we will remove access to the work immediately and investigate your claim 


\title{
Effect of gain nonlinearity in semiconductor lasers
}

\author{
Niels H. Jensen, P. L. Christiansen, and O. Skovgaard \\ Laboratory of Applied Mathematical Physics, The Technical University of Denmark, DK-2800 Lyngby, Denmark
}

(Received 9 February 1988)

\begin{abstract}
Semiconductor lasers are modeled by single-mode rate equations with Langevin noise terms and the influence of nonlinear gain is investigated. For cw operation the probability distribution for the carrier number and the photon number in the laser cavity is obtained. The corresponding $(2+1)$ dimensional Fokker-Planck equation is derived and integrated on an Amdahl VP1100 vector processor. Above threshold the resulting probability density agrees with the rate-equation predictions. The case of high-speed modulation is also considered. The nonlinear gain is found to stabilize the laser.
\end{abstract}

\section{INTRODUCTION}

Semiconductor lasers are modeled by rate equations. Recently it has been shown that inclusion of nonlinearities in the laser-amplification process provides a decisive impact on the modulation properties of the laser. ${ }^{1,2}$ In the present paper we investigate the influence of gain nonlinearities on the noise properties of semiconductor lasers subjected to $\mathrm{cw}$ as well as pulsed operation. We solve numerically the single-mode rate equations with Langevin noise terms. From the resulting time series we obtain the probability distributions which give a good measure of the influence of the nonlinear gain. In the case of $\mathrm{cw}$ operation we also derive the corresponding Fokker-Planck equation and compare numerically obtained stationary solutions to results obtained by direct numerical integration of the rate equations. A numerical algorithm appropriate to obtain stationary solutions of the Fokker-Planck equation has been developed and implemented on an Amdahl VP1100 vector processor.

The paper is organized as follows. Section II presents the laser rate equations with Langevin noise terms (Sec. II A) as well as the derivation of the corresponding Fokker-Planck equation (Sec. II B). In Sec. III we investigate the influence of nonlinear gain on the $\mathrm{cw}$ operation properties (Sec. III A) and we consider the case of pulsed high-speed operation (Sec. III B). Section V contains our conclusion. An appendix is devoted to a detailed description of the algorithm used to solve the Fokker-Planck equation.

\section{SEMICONDUCTOR LASER MODEL}

\section{A. Rate equations}

The semiconductor laser is modeled by the well-known single-mode rate equations with Langevin noise terms:

$$
\begin{aligned}
& \frac{d N}{d t}=\Phi_{N}(N, S)+F_{N}(t), \\
& \frac{d S}{d t}=\Phi_{S}(N, S)+F_{S}(t) .
\end{aligned}
$$

Here $\Phi_{N}$ and $\Phi_{S}$ are the drift coefficients given by

$$
\Phi_{N}(N, S)=J(t)-\frac{N}{\tau_{s}}-G(N, S) S
$$

and

$$
\Phi_{S}(N, S)=\left\{G(N, S)-\frac{1}{\tau_{p}}\right] S+\beta \frac{N}{\tau_{s}},
$$

$G(N, S)$ being the gain function ${ }^{2}$

$$
G(N, S)=G_{N}\left(N-N_{0}\right)(1-\varepsilon S) \text {. }
$$

In (2.1)-(2.3) $N$ and $S$ are the carrier number and the photon number, respectively. $\tau_{s}$ and $\tau_{p}$ are the carrier lifetime and the photon lifetime. $\beta$ is the spontaneousemission factor. $J(t)$ is the injection current which we shall give in terms of the threshold current, $J_{\text {th }}=\left(N_{0}+1 / G_{N} \tau_{p}\right) / \tau_{s}$. In the gain function, (2.3), $G_{N}$ denotes the gain constant, and $N_{0}$ is the carrier number at transparency. $(1-\varepsilon S)$ is a suppression factor, $\varepsilon$ being the gain nonlinearity parameter.

The terms $F_{N}(t)$ and $F_{S}(t)$ in $(2.1)-(2.2)$ are the Langevin noise terms accounting for stochastic fluctuations in $N$ and $S$. Thus we have the mean values ${ }^{3}$

$$
\left\langle F_{N}(t)\right\rangle=\left\langle F_{S}(t)\right\rangle=0 .
$$

The second moments of the Langevin noise terms give the autocorrelations and cross correlations

$$
\begin{aligned}
& \left\langle F_{N}\left(t^{\prime}\right) F_{N}\left(t^{\prime \prime}\right)\right\rangle=2 D_{N N} \delta\left(t^{\prime}-t^{\prime \prime}\right), \\
& \left\langle F_{N}\left(t^{\prime}\right) F_{S}\left(t^{\prime \prime}\right)\right\rangle=2 D_{N S} \delta\left(t^{\prime}-t^{\prime \prime}\right), \\
& \left\langle F_{S}\left(t^{\prime}\right) F_{S}\left(t^{\prime \prime}\right)\right\rangle=2 D_{S S} \delta\left(t^{\prime}-t^{\prime \prime}\right) .
\end{aligned}
$$

The diffusion coefficients, $2 D_{N N}, 2 D_{N S}$, and $2 D_{S S}$, are determined by application of the fluctuation-dissipation theorem ${ }^{4}$ and the Einstein relations between stimulated and spontaneous emission. ${ }^{5}$ From Ref. 6 follow

$$
\begin{aligned}
& 2 D_{N N}(N, S)=J(t)+\frac{N}{\tau_{s}}+\left[2 \beta \frac{N}{\tau_{s}}-G(N, S)\right] S, \\
& 2 D_{N S}(N, S)=-\left[\left(2 \beta \frac{N}{\tau_{s}}-G(N, S)\right] S+\beta \frac{N}{\tau_{s}}\right],
\end{aligned}
$$


$2 D_{S S}(N, S)=\left(2 \beta \frac{N}{\tau_{s}}-G(N, S)+\frac{1}{\tau_{p}}\right) S+\beta \frac{N}{\tau_{s}}$

\section{B. Fokker-Planck equation}

The Fokker-Planck equation associated with the Langevin equations $(2.1)-(2.7)$ is $^{7}$

$$
\frac{\partial \hat{P}}{\partial t}=\mathcal{L}(N, S) \hat{P}
$$

where $\hat{P}=\hat{P}(N, S ; t)$ is the probability density for the values $N$ and $S$ at time $t$. The Fokker-Planck operator $\mathcal{L}(N, S)$ can be expressed in terms of the laser drift and diffusion coefficients as ${ }^{7}$

$$
\begin{aligned}
\mathcal{L}(N, S)= & -\left[\frac{\partial}{\partial N} \Phi_{N}(N, S)+\frac{\partial}{\partial S} \Phi_{S}(N, S)\right] \\
& +\frac{\partial^{2}}{\partial N^{2}} D_{N N}(N, S)+2 \frac{\partial^{2}}{\partial N \partial S} D_{N S}(N, S) \\
& +\frac{\partial^{2}}{\partial S^{2}} D_{S S}(N, S) .
\end{aligned}
$$

Inserting $\Phi_{N}, \Phi_{S}, D_{N N}, D_{N S}$, and $D_{S S}$ we obtain

$$
\begin{aligned}
\frac{\partial \hat{P}}{\partial t}= & D_{N N}(N, S) \frac{\partial^{2} \widehat{P}}{\partial N^{2}}+2 D_{N S}(N, S) \frac{\partial^{2} \widehat{P}}{\partial N \partial S} \\
& +D_{S S}(N, S) \frac{\partial^{2} \widehat{P}}{\partial S^{2}}+D_{N}(N, S) \frac{\partial \hat{P}}{\partial N} \\
& +D_{S}(N, S) \frac{\partial \hat{P}}{\partial S}+D_{0}(N, S) \hat{P},
\end{aligned}
$$

where we have introduced $D_{N}, D_{S}$, and $D_{0}$ as

$D_{N}(N, S)=2 \frac{\partial}{\partial N} D_{N N}(N, S)+2 \frac{\partial}{\partial S} D_{N S}(N, S)-\Phi_{N}(N, S)$,

$D_{S}(N, S)=2 \frac{\partial}{\partial S} D_{S S}(N, S)+2 \frac{\partial}{\partial N} D_{N S}(N, S)-\Phi_{S}(N, S)$,

and

$$
\begin{aligned}
D_{0}(N, S)= & \frac{\partial^{2}}{\partial N^{2}} D_{N N}(N, S)+2 \frac{\partial^{2}}{\partial N \partial S} D_{N S}(N, S) \\
& +\frac{\partial^{2}}{\partial S^{2}} D_{S S}(N, S)-\frac{\partial}{\partial N} \Phi_{N}(N, S) \\
& -\frac{\partial}{\partial S} \Phi_{S}(N, S) .
\end{aligned}
$$

This Fokker-Planck equation for the semiconductor laser appears to be new.

\section{RESULTS}

The system of two ordinary differential equations given by (2.1)-(2.7) is solved numerically for initial conditions obtained as stationary solutions to the rate equations without noise and injection current $J(0)$. For each discrete time value (spaced by $\Delta t^{\prime}$ ) it has been shown in Ref. 6 that the Langevin noise terms can be generated from a two-dimensional Gaussian distribution having zero mean value [cf. (2.4)] and obeying the correlation expressions $(2.5)-(2.7)$. Thus, at the beginning of each time step, the Langevin noise is included by addition of Gaussian noise factors. The noise factors remain constant throughout one time step. The numerical integration results in discrete time series, $N(i)$ and $S(i)$, $i=1,2, \ldots, N_{\mathrm{st}}$. From the time series we deduce the noise statistics.

The remaining part of this section is divided into two subsections. First we investigate the cw operation $[J(t)=$ const $]$ and next we investigate the influence of nonlinear gain on the noise statistics under high-speed (sinusoidal) modulation. Throughout the text we use fixed system parameters chosen as representative figures for GaAs Fabry-Perot semiconductor lasers. The fundamental laser parameters are listed in Table I. For the nonlinear gain parameter we use four values of $\varepsilon(0$, $\left.1 \times 10^{-8}, 1 \times 10^{-7}, 5 \times 10^{-7}\right)$. The value of $\varepsilon$ is given in terms of a cavity volume of $1 \times 10^{-16} \mathrm{~m}^{3}$. In the calculations we use time steps of length $\Delta t^{\prime}=10$ psec.

\section{A. cw operation}

Here the injection current is a constant, $J(t)=J_{b}$. From the time series obtained as described above we obtain the two-dimensional probability density function, $P(N, S)$. The intervals 0 to $\max \{N(i)\}$ and 0 to $\max \{S(i)\}$ are divided into 1000 and 100 intervals, respectively, and the probability distribution is obtained simply by counting the frequency of being in a given box formed by such a grid. The grid parameters (1000 and 100) are chosen in accordance with the relative fluctuations (relative to the mean value) in the carrier number (small fluctuations) and in the photon number (larger fluctuations), respectively. In Figs. 1(a)-1(d) we show our results for the four values of the nonlinearity parameter $\varepsilon\left(0,1 \times 10^{-8}, 1 \times 10^{-7}, 5 \times 10^{-7}\right)$. The injection current is $J_{b}=1.4 J_{\text {th }}$. Results are shown for time series of length $N_{\text {st }}=6000$.

The Fokker-Planck equation (2.9) has a complicated structure. Because the rate equations (2.1)-(2.7) do not satisfy the potential condition, ${ }^{7}$ or equivalently the condition of detailed balance, ${ }^{7}$ no analytical solution to (2.9) may be found, not even a stationary solution $(\partial P / \partial t=0)$. Therefore, in the Appendix we develop a numerical algorithm by which stationary (and in principle dynamical) solutions to the Fokker-Planck equation can be obtained.

TABLE I. Laser parameters.

\begin{tabular}{lll}
\hline \multicolumn{1}{c}{ Parameter } & Symbol & \multicolumn{1}{c}{ Value } \\
\hline Carrier lifetime & $\tau_{s}$ & $3 \mathrm{nsec}$ \\
Photon lifetime & $\tau_{p}$ & $1 \mathrm{psec}$ \\
Gain constant & $G_{N}$ & $1 \times 10^{4} \mathrm{sec}^{-1}$ \\
Gain constant & $N_{0}$ & $1 \times 10^{8}$ \\
Spontaneous-emission factor & $\beta$ & $1 \times 10^{-5}$ \\
Injection current & $J_{b}$ & $1.4 J_{\mathrm{th}}$ \\
\hline \hline
\end{tabular}


The problem is solved as a converging initial-value problem. Referring to Figs. 1(a)-1(d) we apply a Gaussian profile (A6) as initial condition. The standard deviations $\sigma_{x}$ and $\sigma_{y}$ are estimated from the distributions in Figs. 1(a)-1(d). In Table II we have listed the values of $\sigma_{x}$ and $\sigma_{y}$ together with the physical parameters $\sigma_{N}$ and $\sigma_{S}$. In
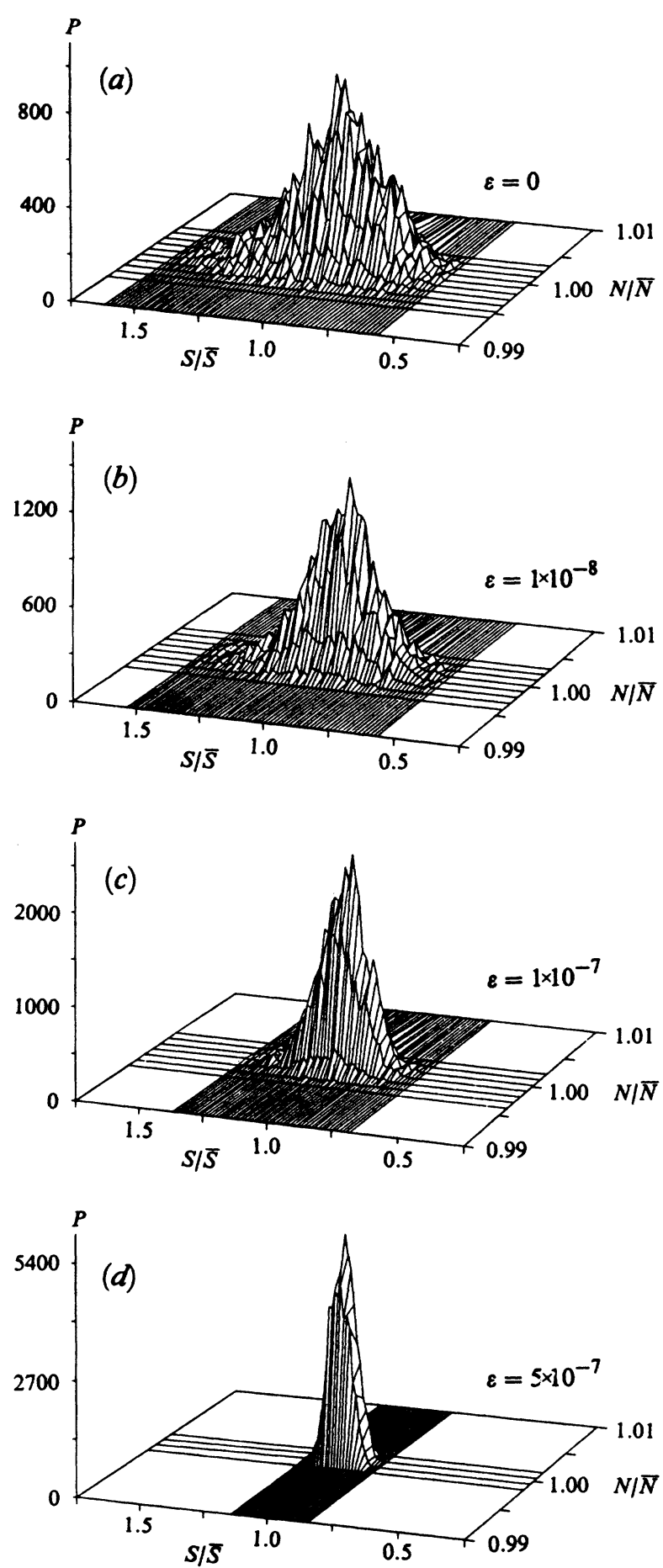

the numerical solution of the Fokker-Planck equation we have used $\gamma_{N}=0.01$ and $\gamma_{S}=0.5$ [see (A1)] and grid parameters $L_{N}=64$ and $L_{S}=64$ [see (A7)]. From preliminary calculations it was shown that the Fokker-Planck operator $\Xi[\mathrm{see}(\mathrm{A} 10 \mathrm{a})]$, assumes values as high as $\pm 10^{13}$ $\mathrm{sec}^{-1}$. As a compromise between stability and minimal
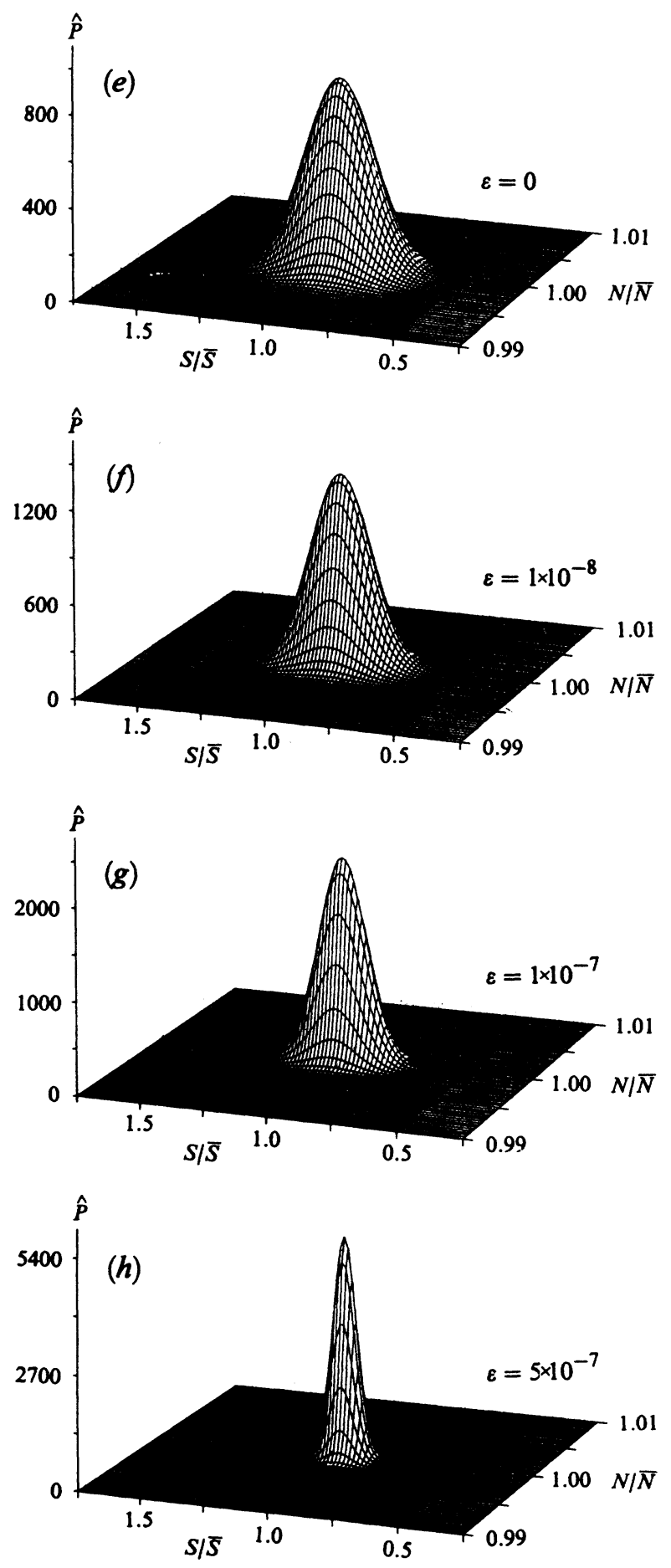

FIG. 1. Two-dimensional probability density functions (a)-(d) obtained from direct numerical integration of the rate equations (2.1)-(2.7), and (e)-(h) as stationary solutions of the Fokker-Planck equation (2.9). The injection current is $J_{b}=1.4 J_{\text {th }}$. Laser parameters as indicated in Table $I$. The values of the gain nonlinearity parameter $\varepsilon$ are (a), (e), $\varepsilon=0 ;(b),(f), \varepsilon=1 \times 10^{-8} ;(\mathrm{c}),(\mathrm{g}), \varepsilon=1 \times 10^{-7}$; (d),(h), $\varepsilon=5 \times 10^{-7}$. 
TABLE II. Estimated input parameters.

\begin{tabular}{lcccc}
\hline \hline $10^{7} \varepsilon$ & $10^{3} \sigma_{x}$ & $10^{3} \sigma_{N} / \bar{N}$ & $10^{3} \sigma_{y}$ & $10^{3} \sigma_{S} / \bar{S}$ \\
\hline 0 & 72 & 1.44 & 134 & 134 \\
0.1 & 55 & 1.10 & 118 & 118 \\
1.0 & 39 & 0.78 & 93 & 93 \\
5.0 & 32 & 0.64 & 47 & 47 \\
\hline \hline
\end{tabular}

CPU consumption, the time step $\Delta t$ in (A12) is chosen as $\Delta t=1 \times 10^{-14} \mathrm{sec}$. Anyway, this small time step still requires a large number of computations. For each time step the algorithm developed requires calculation of $(3+2) L_{S}+3 L_{N}$ fast fourier transformations ${ }^{8}$ [see (A9)] amounting to 512 for the given values of $L_{N}$ and $L_{S}$. For a computation over, say 1000 time steps, this number is raised to 512000 . We have implemented the algorithm (A10)-(A12) on an Amdahl VP1100 vector processor at The Danish Computing Center for Research and Education located at The Technical University of Denmark. For computations we have used a fast-Fourier-transform (FFT) algorithm from the standard SSLII library ${ }^{9}$ (DVRFT1). For comparison a typical calculation was performed on the scalar machine IBM 3081, by means of FFT algorithms from the International Mathematical and Statistical Labraries ${ }^{10}$ (IMSL) (FFT2C, e.g.). The vectorization turned out to yield a gain factor of more than 40 in computing time showing the advantage of using vectorized FFT algorithms. A typical calculation over 1000 time steps required only about 40 CPU sec on the Amdahl VP1100 vector processor. By use of initial conditions obtained as described above, stationary solutions of the Fokker-Planck equation were obtained after less than 1000 steps. However, in order to check and ensure the stability of the solution, the results were taken after these 1000 time steps. In Figs. 1(e)-1(h) we show the numerically obtained stationary solutions of the Fokker-Planck equation for the same four values of the gain nonlinearity parameter $\varepsilon\left(0,1 \times 10^{-8}, 1 \times 10^{-7}\right.$, $5 \times 10^{-7}$ ) as used previously. The constant injection current $J_{b}\left(=1.4 \times J_{\text {th }}\right)$ and the laser parameters are also the same (Table I). The results obtained from direct numerical integration of the rate equations [Figs. 1(a)-1(d)] are in excellent agreement with the results obtained as stationary solutions of the Fokker-Planck equation [Figs. 1(e)-1(h)]. As expected the standard deviations $\sigma_{N}$ and $\sigma_{S}$ (proportional to $\sigma_{x}$ and $\sigma_{y}$ [see (A6)]) in the stationary regime decrease with increasing gain nonlinearity. For nonlinearities of physically relevant order ${ }^{11}$ $\left(\varepsilon \simeq 10^{-7}\right.$, relative to a cavity volume of $\left.1 \times 10^{-16} \mathrm{~m}^{3}\right)$ we find, in contrast to the $\varepsilon=0$ case, that the fluctuations in both the carrier number and the photon number are heavily reduced. The fluctuations are reduced by factors of 2 and 3 in $\sigma_{N}$ and $\sigma_{S}$, respectively, when $\varepsilon$ changes from 0 to $5 \times 10^{-7}$. Thus the nonlinear gain stabilizes the modulation properties of the laser dramatically also in presence of noise.

Above we have used a constant bias $40 \%$ above threshold. However, for application in high-speed modulation properties the laser is biased below or close to threshold and then modulated at high speeds. In such cases the photon number inside the laser cavity is very close to zero. Only the spontaneous emission, which is a totally quantum-mechanical process, ${ }^{12}$ introduces a photon to the cavity. Thus, at bias below or close to threshold the system is described essentially by the spontaneousemission terms $N / \tau_{s}$ and $\beta N / \tau_{s}$ [see (2.1)-(2.2)]. In Figs. $2(a)-2(d)$ we show results obtained for the same four values of the nonlinearity parameter $\varepsilon$ and the same laser parameters as used before. Here the injection current is $J_{b}=1.04 J_{\text {th }}$, i.e., $4 \%$ above threshold. We see
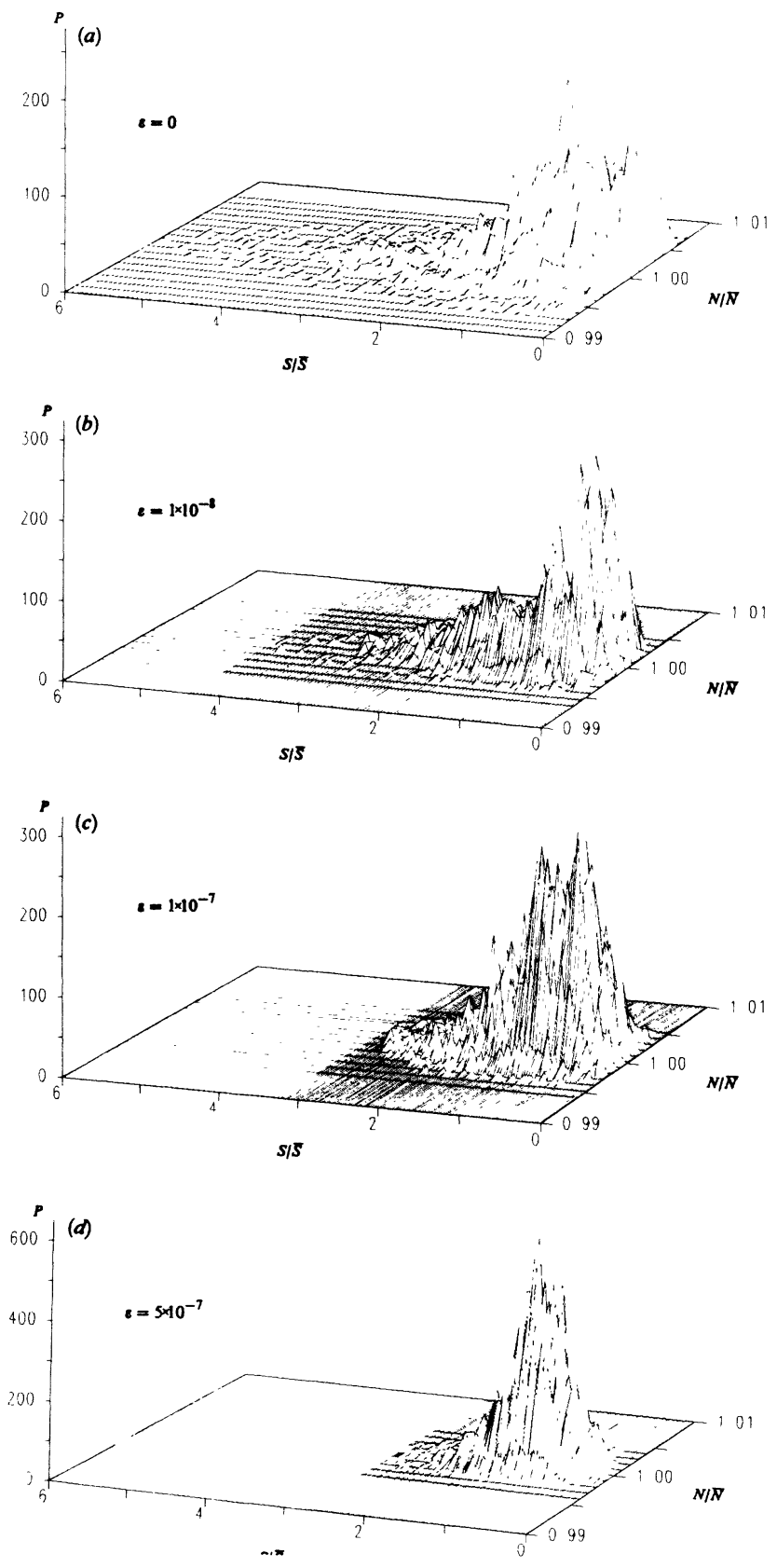

FIG. 2. Two-dimensional probability density functions obtained from direct numerical integration of the rate equations (2.1)-(2.7). The injection current is $J_{b}=1.04 J_{t h}$. Laser parameters as indicated in Table $I$. The values of the gain nonlinearity parameter $\varepsilon$ are (a), $\varepsilon=0$; (b), $\varepsilon=1 \times 10^{-8}$; (c), $\varepsilon=1 \times 10^{-7}$; (d), $\varepsilon=5 \times 10^{-7}$. 
that the nonlinear gain in this case also stabilizes the dynamics. For small $\varepsilon\left(\varepsilon<10^{-7}\right)$ the probability does not look Gaussian as was the case for operation well above threshold. This is because noise events, strictly speaking, can only change the number of photons at increasing rates. For higher values of the gain nonlinearity, the nonlinear gain has stabilized the system to yield Gaussian-looking profiles [Fig. 2(d)] due to strong damping imposed by the gain nonlinearity. For bias at $4 \%$ above threshold the stationary solution to Eq. (2.8) is not Gaussian in the $S$ direction. As a consequence a more complicated algorithm (not based on periodic boundary conditions) must be applied to obtain stationary solutions.

\section{B. Pulsed operation}

In Sec. III A we have investigated the influence of gain nonlinearities on the $\mathrm{cw}$ operational properties of the semiconductor-laser model (2.1)-(2.7). In this section we investigate the influence of gain nonlinearities on highspeed pulse modulation of the laser. For simplicity we use a sinusoidal modulation function given as

$$
J(t)=J_{b}+J_{p} \sin \left(2 \pi f_{m} t\right),
$$

where the constant bias term $J_{b}$ and the modulation amplitude $J_{p}$ are given in terms of the threshold current $J_{\text {th }}$. In (3.1), $f_{m}$ is the modulation frequency, $f_{m}=1 /$ $\left(N_{p} \Delta t^{\prime}\right), N_{p}$ being the number of discrete time steps of length $\Delta t^{\prime}$ in a modulation period.

In order to take account of the low-pass filtering in a practical receiver, we use a digital filter with the following impulse response:

$$
h(\alpha)=\left\{\begin{array}{l}
\sin ^{2}(\pi \alpha / K), \quad \alpha=0,1, \ldots, K \\
0, \text { elsewhere }
\end{array}\right.
$$

which has the transfer function

$H(f)=\frac{\frac{1}{2} K \Delta t^{\prime}}{1-\left(f K \Delta t^{\prime}\right)^{2}} \frac{\sin \left(\pi f K \Delta t^{\prime}\right)}{\pi f} \exp \left(-j \pi f K \Delta t^{\prime}\right)$.

A convolution of the "raw" output data obtained numerically from the rate equations with $h(\alpha)$ corresponds to a multiplication by $H(f)$ in the frequency domain, thus yielding the desired low-pass filtering. The sampling number $K$ in (3.2) is determined such that the 3 -dB frequency of $|H(f)|^{2}$ corresponds to the modulation frequency. In the following calculations we have used $N_{p}=67$ and $\Delta t^{\prime}=10$ psec yielding $f_{m}=1.49 \mathrm{GHz}$. From (3.2) it then follows that $K=48$. Each pulse is thus averaged and weighted according to (3.2), yielding a vector containing the weighted pulse values (only the statistics for the photon number will be considered here). The peak values are obtained at time slots corresponding to peak values obtained in a dynamical simulation without noise. From this vector we deduce the noise statistics: The interval from zero to maximum of smoothed (weighted) peak values is divided into 100 intervals and the density of peaks in each interval is obtained to give the prob-
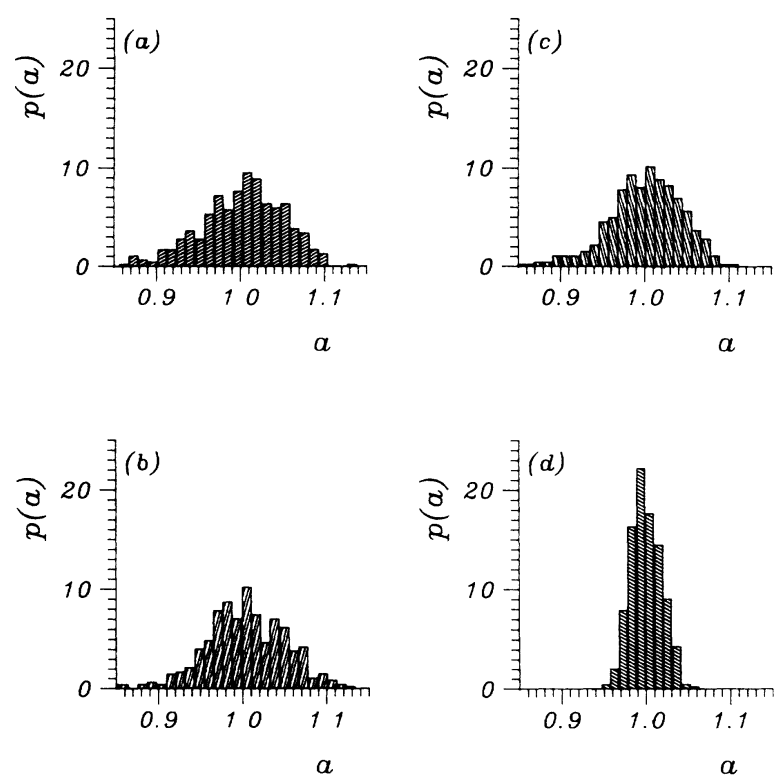

FIG 3. Probability of the peak values of the photon-pulse response in the case of $1.49 \mathrm{GHz}$ sinusoidal modulation. $J_{b}=1.3 J_{\text {th }}$ and $J_{p}=0.25 J_{\mathrm{th}}$. Laser parameters are given in Table I. The values of the gain nonlinearity parameter $\varepsilon$ are (a), $\varepsilon=0$; (b), $\varepsilon=1 \times 10^{-8}$; (c), $\varepsilon=1 \times 10^{-7}$; (d), $\varepsilon=5 \times 10^{-7}$.

ability density function.

Our aim is to examine the relative probability distribution $p(a)$, where $a$ is the (smoothed) peak value relative to the mean value of peaks. Thus $\bar{a}=1$. In order to obtain reasonable statistics (a reasonably large number of pulses) the number of time steps was chosen as 30000 corresponding to 447 full modulation periods. Initial data were taken as stationary solutions to the noise-free rate equations $\left(F_{N}=F_{S}=0\right)$ and bias $J_{b}$. Because stationary solutions do not satisfy a dynamical solution to the rate equations there will be some transients. In order to neglect pulses in the transient region we neglect the first 27 pulses (which were proven sufficient) and perform the statistics on the last 420 pulses only.

In Fig. 3 we show our results obtained for the same parameters as used before. The current parameters are $J_{b}=1.3 J_{\text {th }}$ and $J_{p}=0.25 J_{\text {th }}$. We emphasize that (3.1) with these current parameters corresponds to a first-order approximation to a 50\%-duty-cycle square-wave modulation picture with current switched between $1.1 J_{\text {th }}$ and $1.5 J_{\text {th }}$ (approximately).

As the gain nonlinearity is increased we observe a clear narrowing in the distribution. This shows that the nonlinear gain in the case of pulsed operation also suppresses the fluctuations and reduces the influence of noise.

\section{CONCLUSION}

We have examined the influence of nonlinear gain on the single-mode semiconductor-laser rate equations with Langevin noise terms. For both $\mathrm{cw}$ and pulsed operation we found that inclusion of nonlinear gain terms in the rate equations imposes stabilizing effects on the dynamics 
thus showing the importance of such terms. In the case of cw operation we derived the corresponding FokkerPlanck equation. This equation which appeared to be new was solved numerically by use of a developed finitedifference-pseudospectral algorithm. Very good agreement between results obtained from direct numerical integration of the rate equations and from solutions of the Fokker-Planck equation was found.

\section{ACKNOWLEDGMENTS}

B. Tromborg and F. If are thanked for valuable suggestions and discussions

\section{APPENDIX: NUMERICAL SOLUTION OF THE FOKKER-PLANCK EQUATION}

The Fokker-Planck equation (2.9) is a $(2+1)$ dimensional partial differential equation. For proper boundary and initial conditions we shall obtain stationary solutions and expect solutions comparable to the distributions obtained by direct numerical integration of the laser rate equations. From these results we expect $\widehat{P}$ to be zero except in a limited region near the mean values $\bar{N}$ and $\bar{S}$. We thus examine a two-dimensional region around the mean values and apply periodic boundary conditions

$$
\begin{aligned}
\hat{P}\left(\left(1-\alpha_{N} \gamma_{N}\right) \bar{N}, S ; t\right)=\hat{P}\left(\left(1+\alpha_{N} \gamma_{N}\right) \bar{N}, S ; t\right), \\
\left(1-\gamma_{S}\right) \bar{S} \leq S \leq\left(1+\gamma_{S}\right) \bar{S}
\end{aligned}
$$

$$
\begin{aligned}
\hat{P}\left(N,\left(1-\alpha_{S} \gamma_{S}\right) \bar{S} ; t\right)= & \hat{P}\left(N,\left(1+\alpha_{S} \gamma_{S}\right) \bar{S} ; t\right), \\
& \left(1-\gamma_{N}\right) \bar{N} \leq N \leq\left(1+\gamma_{N}\right) \bar{N}
\end{aligned}
$$

for all values of $\alpha_{N}$ and $\alpha_{S}$ between zero and unity. The parameters $\gamma_{N}$ and $\gamma_{S}$ are fixed constants less than 1 .

As an initial condition we use a two-dimensional Gaussian distribution

$$
\widehat{P}(N, S ; 0) \propto \exp \left[-\frac{(N-\bar{N})^{2}}{2 \sigma_{N}^{2}}-\frac{(S-\bar{S})^{2}}{2 \sigma_{S}^{2}}\right] .
$$

In order to apply a Fourier spectral method ${ }^{12}$ to compute the partial derivatives in the $N$ and $S$ coordinates we simplify the problem by the introduction of new variables

$$
x=\frac{N-\left(1-\gamma_{N}\right) \bar{N}}{2 \gamma_{N} \bar{N}}, \quad y=\frac{S-\left(1-\gamma_{S}\right) \bar{S}}{2 \gamma_{S} \bar{S}} .
$$

The Fokker-Planck equation (2.9) is transformed into

$$
\begin{aligned}
\frac{\partial \hat{u}}{\partial t}= & D_{x x}(x, y) \frac{\partial^{2} \hat{u}}{\partial x^{2}}+2 D_{x y}(x, y) \frac{\partial^{2} \hat{u}}{\partial x \partial y}+D_{y y}(x, y) \frac{\partial^{2} \hat{u}}{\partial y^{2}} \\
& +D_{x}(x, y) \frac{\partial \hat{u}}{\partial x}+D_{y}(x, y) \frac{\partial \hat{u}}{\partial y}+D(x, y) \hat{u}
\end{aligned}
$$

where $\widehat{u}=\widehat{u}(x, y ; t)=\widehat{P}(N, S ; t)$ and

$$
\begin{aligned}
& D_{x x}(x, y)=\frac{D_{N N}(N, S)}{\left(2 \gamma_{N} \bar{N}\right)^{2}}, \\
& D_{x y}(x, y)=\frac{D_{N S}(N, S)}{4 \gamma_{N} \gamma_{S} \bar{N} \bar{S}}, \\
& D_{y y}(x, y)=\frac{D_{S S}(N, S)}{\left(2 \gamma_{S} \bar{S}\right)^{2}} ; \\
& D_{x}(x, y)=\frac{D_{N}(N, S)}{2 \gamma_{N} \bar{N}}, \\
& D_{y}(x, y)=\frac{D_{S}(N, S)}{2 \gamma_{S} \bar{S}}, \\
& D(x, y)=D_{0}(N, S) .
\end{aligned}
$$

The boundary conditions (A1) and the initial conditions (A2) become

$$
\begin{array}{ll}
\hat{u}\left(\alpha_{N}, y ; t\right)=\hat{u}\left(1-\alpha_{N}, y ; t\right), & 0 \leq y \leq 1 \\
\hat{u}\left(x, \alpha_{S} ; t\right)=\hat{u}\left(x, 1-\alpha_{S} ; t\right), & 0 \leq x \leq 1
\end{array}
$$

and

$$
\begin{gathered}
\hat{u}(x, y ; 0) \propto \exp \left[-\frac{\left(x-\frac{1}{2}\right)^{2}}{2 \sigma_{x}^{2}}-\frac{\left(y-\frac{1}{2}\right)^{2}}{2 \sigma_{y}^{2}}\right], \\
\sigma_{x}=\frac{\sigma_{N}}{2 \gamma_{N} \bar{N}}, \quad \sigma_{y}=\frac{\sigma_{S}}{2 \gamma_{S} \bar{S}} .
\end{gathered}
$$

which clearly satisfy (A5).

In order to solve (A4) subjected to the boundary condition (A5) and the initial condition (A6) we use a Fourier spectral method ${ }^{12}$ in the $x$ and $y$ coordinates and a finitedifference approximation in time $t$, i.e., a combined finite-difference-pseudospectral method. We discretize the unit square $\left[0,1\left[\times\left[0,1\left[\right.\right.\right.\right.$ in $L_{N}$ and $L_{S}$ mesh points as follows:

$$
\begin{aligned}
& x_{k}=\frac{(k-1)}{L_{N}}, \quad k=1,2, \ldots, L_{N} \\
& y_{l}=\frac{(l-1)}{L_{S}}, \quad l=1,2, \ldots, L_{S} .
\end{aligned}
$$

For a fixed value of $l$ (and fixed time $t$ ) we introduce

$$
\begin{aligned}
& V_{L_{N}}\left(x_{k}\right)=\hat{u}\left(x_{k}, y_{l} ; t\right), \\
& \qquad l, t \text { fixed }, k=1,2, \ldots, L_{N} .
\end{aligned}
$$

The discrete Fourier transform of the continuous function $\hat{u}$ for fixed $y$ (corresponding to $y_{l}$ ) and fixed $t$ thus has the form ${ }^{13}$

$$
\begin{array}{r}
V_{L_{N}}\left(x_{k}\right)=\sum_{n=1}^{L_{N}} a_{n} \exp \left[-2 \pi i \frac{(k-1)}{L_{N}} n\right], \\
k=1,2, \ldots, L_{N}
\end{array}
$$

where 


$$
\begin{array}{r}
a_{n}=\frac{1}{L_{N}} \sum_{k=1}^{L_{N}} V_{L_{N}}\left(x_{k}\right) \exp \left[\begin{array}{r}
2 \pi i \frac{(n-1)}{L_{N}} k \\
n=1,2, \ldots, L_{N}
\end{array}\right.
\end{array}
$$

where $i$ is the imaginary unit.

Let $\mathcal{F}_{N}$ denote the expansion in $x$ space (A9b) and $\mathcal{F}_{N}^{-1}$ denote the inverse expansion (A9a). Similarly we define $\mathscr{F}_{S}$ and $\mathcal{F}_{S}^{-1}$ for the corresponding expansions in $y$ space.
We thus write the discritized form of the Fokker-Planck equation as

$$
\begin{aligned}
\frac{\partial \widehat{u}\left(x_{k}, y_{l} ; t\right)}{\partial t} & =\Xi\left(x_{k}, y_{l} ; t\right), \\
k & =1,2, \ldots, L_{N}, l=1,2, \ldots, L_{S}
\end{aligned}
$$

where the right-hand side is given by

$$
\begin{aligned}
\Xi\left(x_{k}, y_{l} ; t\right)= & D_{x x}\left(x_{k}, y_{l}\right)\left(\mathcal{F}_{N}^{-1}\left\{-k_{n}^{2} \mathcal{F}_{N}\left[V_{L_{N}}\left(x_{k}\right)\right]\right\}_{l=1,2, \ldots, L_{S}}\right) \\
& +2 D_{x y}\left(x_{k}, y_{l}\right)\left\{\mathcal{F}_{N}^{-1}\left[i k_{n} \mathcal{F}_{N}\left(\mathcal{F}_{S}^{-1}\left\{i k_{m} \mathcal{F}_{S}\left[V_{L_{S}}\left(y_{l}\right)\right]\right\}_{k=1,2, \ldots, L_{N}}\right)\right]_{l=1,2, \ldots, L_{S}}\right\} \\
& +D_{y y}\left(x_{k}, y_{l}\right)\left(\mathcal{F}_{S}^{-1}\left\{-k_{m}^{2} \mathcal{F}_{S}\left[V_{L_{S}}\left(y_{l}\right)\right]\right\}_{k=1,2, \ldots, L_{N}}\right) \\
& +D_{x}\left(x_{k}, y_{l}\right)\left(\mathcal{F}_{N}^{-1}\left\{-i k_{n} \mathcal{F}_{N}\left[V_{L_{N}}\left(x_{k}\right)\right]\right\}_{l=1,2, \ldots, L_{S}}\right) \\
& +D_{y}\left(x_{k}, y_{l}\right)\left(\mathcal{F}_{S}^{-1}\left\{-i k_{m} \mathcal{F}_{S}\left[V_{L_{S}}\left(y_{l}\right)\right]\right\}_{k=1,2, \ldots, L_{N}}\right) \\
& +D\left(x_{k}, y_{l}\right) \hat{u}\left(x_{k}, y_{l} ; t\right), \quad k=1,2, \ldots, L_{N}, l=1,2, \ldots, L_{S} .
\end{aligned}
$$

In (A10) we have introduced the wave numbers ${ }^{13}$

$$
\begin{aligned}
& k_{n}=\left\{\begin{array}{l}
2 \pi(n-1), \quad n=1,2, \ldots, L_{N} / 2 \\
0, \quad n=L_{N} / 2+1 \\
2 \pi\left[L_{N}-(n-1)\right], \quad n=L_{N} / 2+2, L_{N} / 2+3, \ldots, L_{N}
\end{array}\right. \\
& k_{m}= \begin{cases}2 \pi(m-1), \quad m=1,2, \ldots, L_{S} / 2 \\
0, & m=L_{S} / 2+1 \\
2 \pi\left[L_{S}-(m-1)\right], & m=L_{S} / 2+2, L_{S} / 2+3, \ldots, L_{S}\end{cases}
\end{aligned}
$$

which reflect the periodic boundary conditions (A5).

For the derivative in time we discretize the time $t$ in $t_{j}=j \Delta t, j=0,1, \ldots$, and apply a second-order finitedifference approximation. Thus the final algorithm becomes

$$
\begin{gathered}
\widehat{u}\left(x_{k}, y_{l} ; t_{j+1}\right)=\widehat{u}\left(x_{k}, y_{l} ; t_{j-1}\right)+2 \Delta t \Xi\left(x_{k}, y_{l} ; t_{j}\right), \\
k=1,2, \ldots, L_{N}, l=1,2, \ldots, L_{S}, j \geq 1
\end{gathered}
$$

For the first time step we use a first-order finite-difference approximation

$\widehat{u}\left(x_{k}, y_{l} ; t_{1}\right)=\widehat{u}\left(x_{k}, y_{l} ; t_{0}\right)+\Delta t \Xi\left(x_{k}, y_{l} ; t_{0}\right)$,

$$
k=1,2, \ldots, L_{N}, l=1,2, \ldots, L_{S} .
$$

${ }^{1}$ G. P. Agrawal, Appl. Phys. Lett. 49, 1013 (1986).

${ }^{2}$ N. H. Jensen, P. L. Christiansen, and O. Skovgaard, IEE Proc.

$\mathrm{J}$ : Optoelectron. (to be published).

${ }^{3}$ M. Lax, Rev. Mod. Phys. 38, 541 (1966).

${ }^{4}$ L. D. Landau and E. M. Lifshitz, Statistical Physics, 3rd ed. (Pergamon, Oxford, 1980), Pt. 1.

${ }^{5}$ A. Yariv, Quantum Electronics, 2nd ed. (Wiley, New York, 1975).

${ }^{6}$ N. H. Jensen, K. E. Stubkjaer, and H. Olesen, IEEE J. Quantum Electron QE-23, 71 (1987)

${ }^{7}$ H. Risken, The Fokker-Planck Equation (Springer-Verlag, Berlin, 1984).

${ }^{8}$ D. F. Elliott and K. A. Rao, Fast Fourier Transforms, Algo- rithms, Analyses, Applications (Academic, New York, 1982).

${ }^{9}$ Amdahl VP/ADS, SSLII-Users Guide (MC-142014-002) (Fujitu Ltd., USA, 1986).

${ }^{10}$ International Mathematical and Statistical Libraries, ed. 9.2 (IMSL Inc., Houston, 1984).

${ }^{11}$ R. S. Tucker and I. P. Kaminow, J. Lightwave Technol. LT-2, 385 (1984).

${ }^{12}$ R. G. Voigt, D. Gottlieb, and Y. Hussaini, Spectral Methods for Partial Differential Equations, (Society for Industrial and Applied Mathematics, Philadelphia, 1984).

${ }^{13} \mathrm{~F}$. If, Technical University of Denmark, The Danish Center for Applied Mathematics and Mechanics, Report No. S31, 1986 (unpublished). 

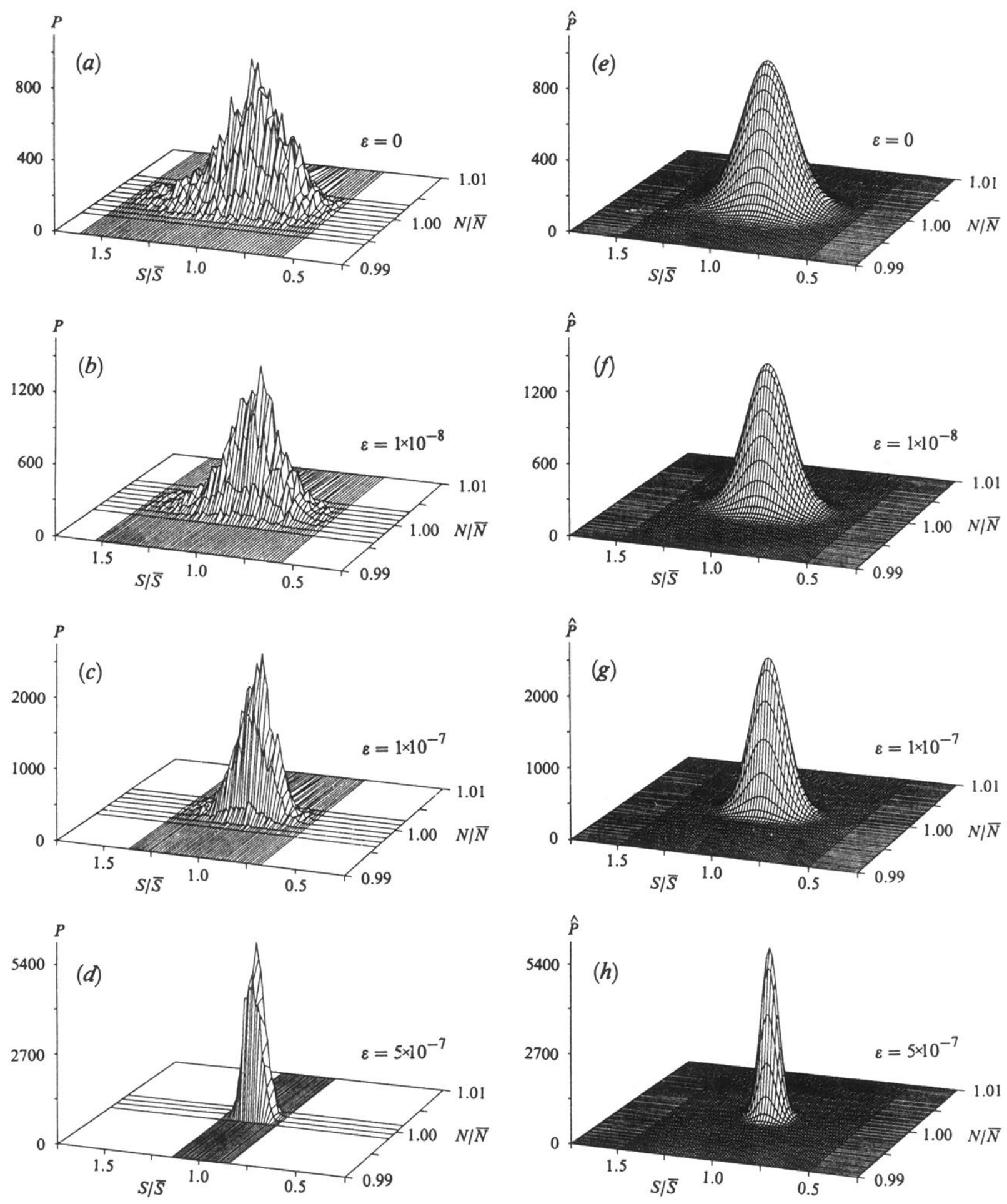

FIG. 1. Two-dimensional probability density functions (a)-(d) obtained from direct numerical integration of the rate equations (2.1)-(2.7), and (e)-(h) as stationary solutions of the Fokker-Planck equation (2.9). The injection current is $J_{b}=1.4 J_{\text {th }}$. Laser parameters as indicated in Table I. The values of the gain nonlinearity parameter $\varepsilon$ are (a), (e), $\varepsilon=0 ;(b),(f), \varepsilon=1 \times 10^{-8} ;(\mathrm{c}),(\mathrm{g}), \varepsilon=1 \times 10^{-7}$; (d), (h), $\varepsilon=5 \times 10^{-7}$. 

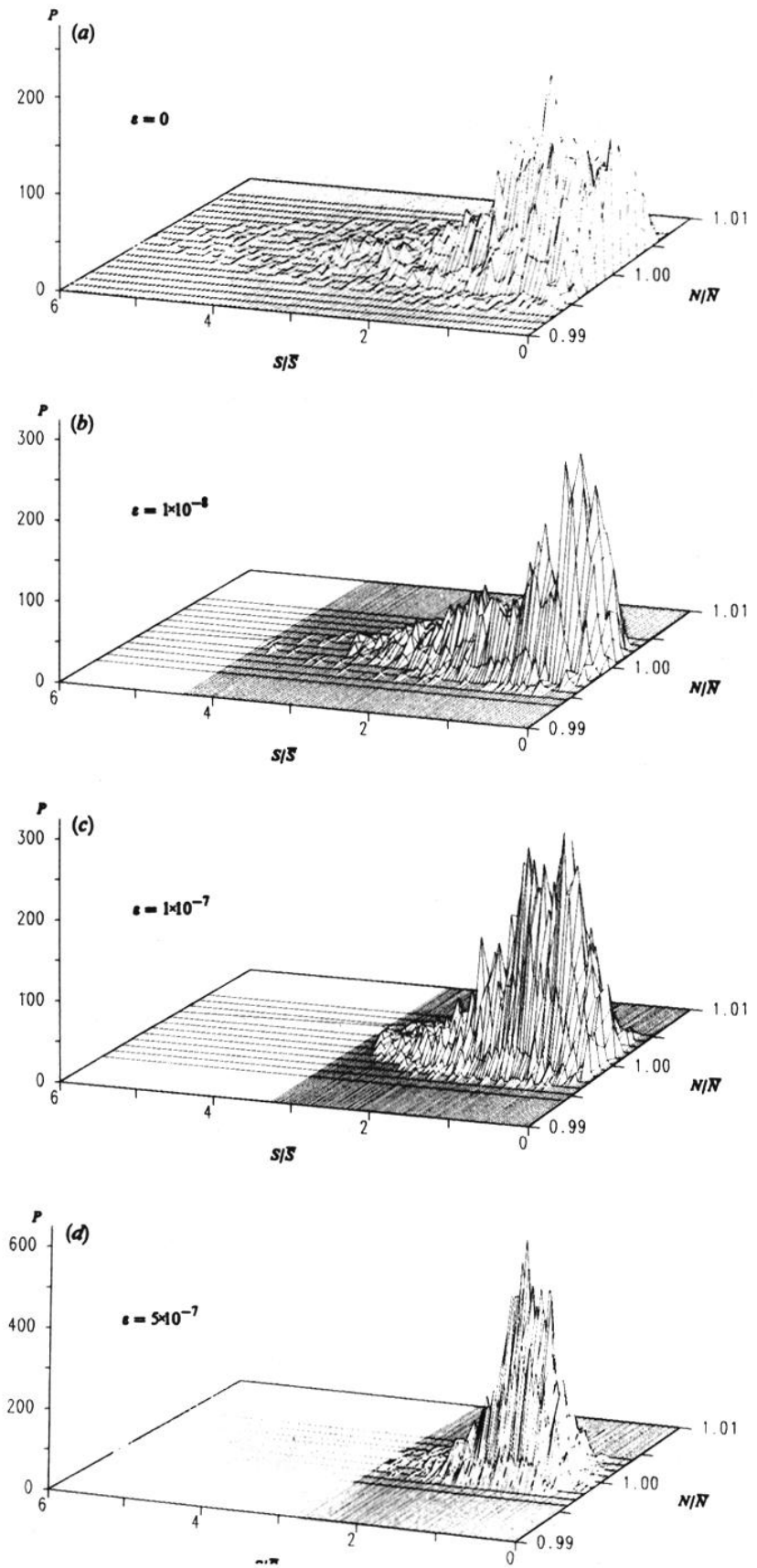

FIG. 2. Two-dimensional probability density functions obtained from direct numerical integration of the rate equations (2.1)-(2.7). The injection current is $J_{b}=1.04 J_{\text {th }}$. Laser parameters as indicated in Table $I$. The values of the gain nonlinearity parameter $\varepsilon$ are (a), $\varepsilon=0$; (b), $\varepsilon=1 \times 10^{-8}$; (c), $\varepsilon=1 \times 10^{-7}$; (d), $\varepsilon=5 \times 10^{-7}$. 